\title{
SUMMARY/PROGRESS REPORT
}

Project Number: 54585

Project Title: Permanganate Treatment of DNAPLs in Reactive Barriers and Source Zone Flooding Schemes

Publication Date: June 30, 2000

Lead Principal Investigator: Dr. Frank W. Schwartz, The Ohio State University, Columbus, OH, frank@geology.ohio-state.edu

Co-Investigator: Dr. Hubao Zhang, Duke Engineering and Services Inc., Albuquerque, NM, hznang@dukeenineering.com

Number of Graduate Students: 3 (1 post-doc and 2 graduate students)

Research Objective: In situ oxidation with potassium permanganate has been widely evaluated, as a potential remediation method for dissolved or pure DNAPL in groundwater system. The goals of this study are (1) to elucidate the basic mechanisms by which potassium permanganate oxidizes common chlorinated solvents, various constituents in aqueous solution, and porousmedium solids, and (2) to assess the potential for chemical oxidation by potassium permanganate to serve as a remedial scheme involving either source zone flooding or reactive barriers. The study is organized with a laboratory component that looks generally at the basic reaction processes and kinetics, and a theoretical component that is developing modeling tools appropriate for designing systems under field conditions.

Research Progress and Implications: This report summarizes work after 3.5 years of a 4-year project. The work mainly consists of six parts. Part (1) is a fundamental study of kinetic reactions between permanganate and five chlorinated ethylenes including PCE, TCE, and three isomers of DCE. Rate parameters were estimated in a range of $\mathrm{pH} 4-8$. Permanganate consumption by other dissolved organic compounds was also estimated for field applications to predict the TCE degradation rate in a system involving multiple contaminants. Part (2) involves series of kinetic experiments conducted to identify products and to parametize the oxidation kinetics in an attempt to assess the utility of this reaction as the basis for the in-situ remediation of groundwater contaminated by chlorinated ethenes. Based on our understanding of various processes involved with TCE oxidation, a kinetic model was developed and the model parameters were estimated.

The kinetic model proposed in this study successfully simulates observed experimental data. Part (3) is an effort to increase the reaction rate of permanganate oxidation by means of phasetransfer-catalysis. Phase transfer catalysts (PTC) work by transferring permanganate ion into the nonaqueous phase where it initiates oxidation decomposition. Selected PTCs increased the rate of TCE decomposition by catalyzing permanganate oxidation in the organic phase. This study suggests that there is significant potential for testing this scheme under field conditions. Part (4) of study examined the influences of pentyltriphenylphosphonium as a PTC on the rate of permanganate oxidation of DNAPLs (TCE, PCE, TCA, and TECA) in pure phases and mixtures. TCA and TECA appeared to increase the permanganate consumption significantly when then were mixed with TCE or PCE. The increase in consumption rates with the PTC was most 
remarkable with the mixtures of PCE and TCA. The relative abundance of each component in the various mixtures determines the extent of enhancements in DNAPL oxidations (Figure 1). Another aspect of the study investigated efficiency problems related to permanganate oxidation schemes. Those problems cover competitive permanganate utilization in aquifer materials, manganese dioxide precipitation around zones of high DNAPL, and permeabilityrelated flow bypassing. The results show a dramatic consumption of permanganate by aquifer materials (Figure 2). Mass removal rates are also greatly influenced by manganese dioxide precipitation and flow bypassing (Figure 3). It is anticipated that in actual field settings, the issue of flushing efficiency needs to be considered in the design. A theoretical

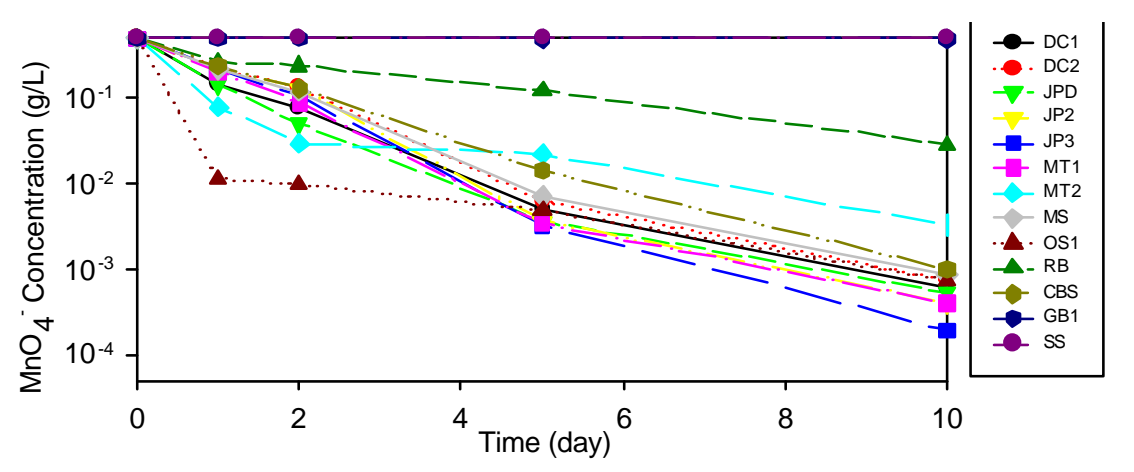

Figure 2. Utilization of permanganate through oxidation of various aquifer materials

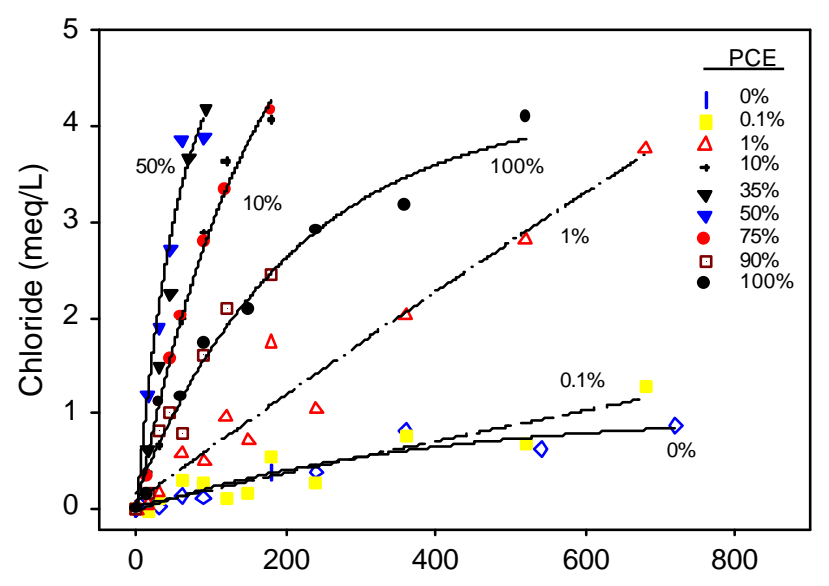

Figure 1. Chloride production from oxidation of PCE /TCA catalyzed by PTPP. The volumetric percentage of PCE in the mixtures varied from 0 to $100 \%$.

framework for the chemical and physical processes was created in part (6). A computer model was developed to simulate the coupled processes of DNAPL dissolution, chemical reaction, and solute mass transport in the in-situ chemical oxidation scheme. The capabilities of this code was tested by comparison with column, test cell, and field experiments. The simulated results matched well with experimental measurements. The computer model provides a useful tool for assisting with the design and the prediction of the oxidation processes under field conditions.

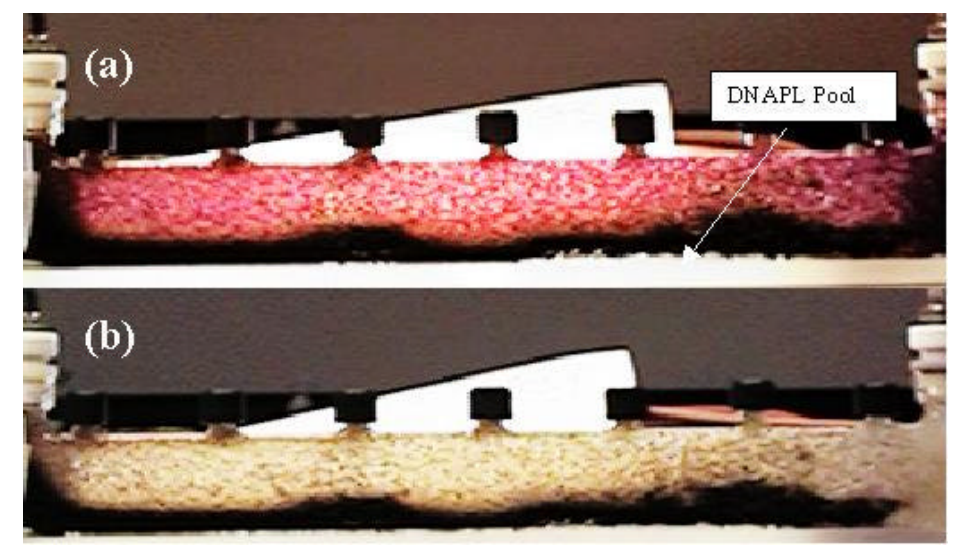

Figure 3. digital images of permanganate flooding of a small glass flow tank. Panel (a) shows conditions after 2 days of flooding. Panel (b) shows conditions 5 hours after flooding stopped. The TCE was sequestered as a rind of precipitate forms and permanganate bypassed this zone. 
Planned Activities: (1) Further research includes detail investigation on mechanisms of permanganate oxidation in nonaqueous phase, cosolvent assisted permanganate oxidation of dissolved DNAPLs, autocatalytic reaction kinetics, and the limitations of permanganate-based remediation scheme. (2) Large tank experiment is in progress to undertake proof of concept studies to determine the efficacy of DNAPL removal in large scale flooding schemes, to elucidate how the efficiency of DNAPL removal is influences by flow by-passing due to multiphase effects and the presence of reaction products, and to develop further promising electrical and optical monitoring schemes.

Information Access: 10 papers have been prepared or submitted. The results of all these efforts and published references are presented in a 69 page comprehensive report entitled "Permanganate Treatment of DNAPLs in Reactive Barriers and Source Zones", which is available at www.geology.ohio-state.edu/ hydro or also from the PI. 